\title{
A study of the oxepane synthesis by a 7-endo electrophile-induced cyclization reaction of alkenylsulfides. An approach towards the synthesis of septanosides
}

\author{
Andrea Köver, M. Isabel Matheu, Yolanda Díaz,* and Sergio Castillón* \\ Departament de Química Analítica i Química Orgànica, Universitat Rovira i Virgili, \\ C/ Marcel.li Domingo s/n 43007, Tarragona, Spain. \\ E-mail:sergio.castillon@urv.net,yolanda.diaz@urv.net
}

\section{Dedicated to the Professor Joan Bosch at occasion of his 60th birthday}

\begin{abstract}
A procedure for the stereoselective synthesis of 2-deoxy-2-iodo-septanosides from pyranoses is reported. The procedure involves two reactions: Wittig-Horner olefination to give alkenyl sulfanyl derivatives, and electrophilic iodine-induced cyclization to give phenyl 2-deoxy2-iodo-1-thio-septanosides (20) or 2-deoxy-2-iodo-septanosides (26a,b), in this case by subsequent hydrolysis of a phenylsulfanyl group under the reaction conditions. The seven membered ring of septanosides was only formed in moderate to low yields, preferably through a 7-endo cyclization, when an isopropylidene group was present as protecting group. The use of benzyl groups as protecting moieties in the pyranose does not afford the septanoside ring. However, when the reactions conditions were forced using more basic media, the furanoside derivatives 3 were obtained.
\end{abstract}

Keywords: Oxepane, septanosides, 7-endo, electrophilic cyclization, 2-deoxy-carbohydrates

\section{Introduction}

Septanosides are ring expanded analogues of pyranosides containing a seven membered ring. ${ }^{1}$ It has been previously demonstrated that septanoside derivatives bind concanavalin $\mathrm{A},{ }^{2}$ are glycosidase inhibitors, ${ }^{3}$ as well as their aza derivatives, ${ }^{4}$ and have been used to define new types of protein-carbohydrate interactions. ${ }^{5}$ Septanoses have been prepared by ring expansion of uloses, by Baeyer-Villiger oxidation of inositols and by Baeyer-Fischer reaction of sugar dialdehydes. ${ }^{1}$ Recently, 1,2-anhydro-septanose derivatives (glycals) have been synthesized using ring closing metathesis ${ }^{6}$ or by cyclization of alkynols induced by a tungsten catalyst. ${ }^{7}$ These $1,2-$ anhydro-septanose derivatives have been used to prepare new septanoside derivatives, ${ }^{8}$ and 
disaccharides containing septanoses. ${ }^{9}$ Septanosides have also been prepared by acid-catalyzed cyclization of hydroxy-acetals. $^{10}$

Recently we reported ${ }^{11}$ a procedure for the synthesis of phenyl 2-deoxy-2-iodo-1-thiopyranosyl glycosides ${ }^{12,13}$ from pentoses through a two step procedure consisting of WittigHorner olefination to give the sulfanylalkenes, and iodonium species-induced cyclization to give the 2-deoxy-2-iodo-1-thiopyranosyl glycosides (Scheme 1).

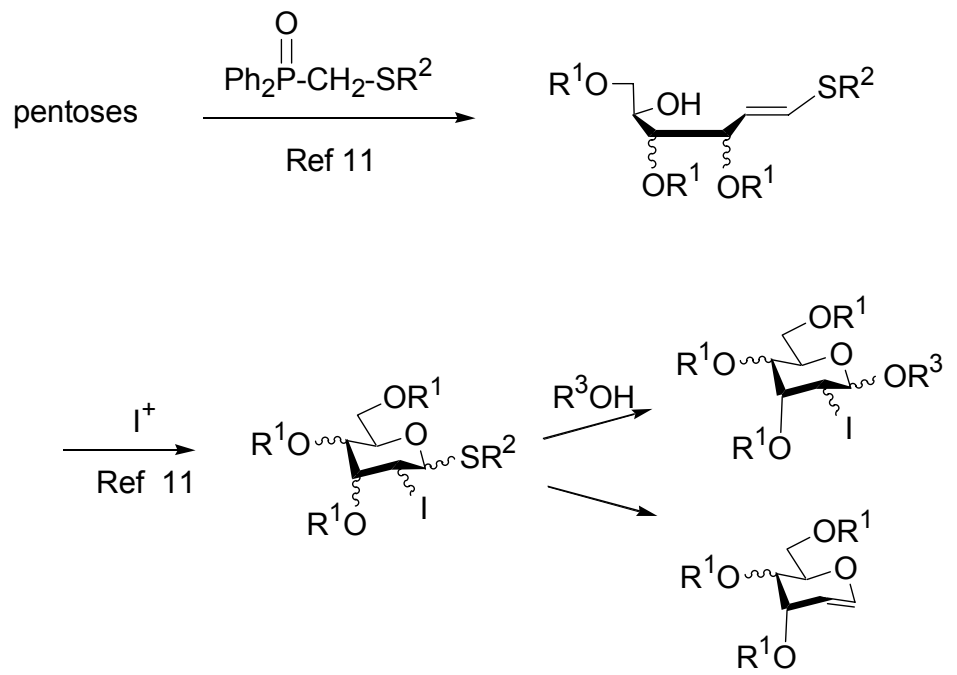

\section{Scheme 1}

These 1-thio-pyranosides were useful glycosyl donors for the stereocontrolled synthesis of 2deoxy-2-iodo-disaccharides. This procedure was particularly efficient for the synthesis of 2deoxy- $\beta$-hexo-glycosides of allo or gulo configuration. ${ }^{11} 1$-Thio-glycosides were also efficiently transformed to glycals ${ }^{14}$ (Scheme 1).

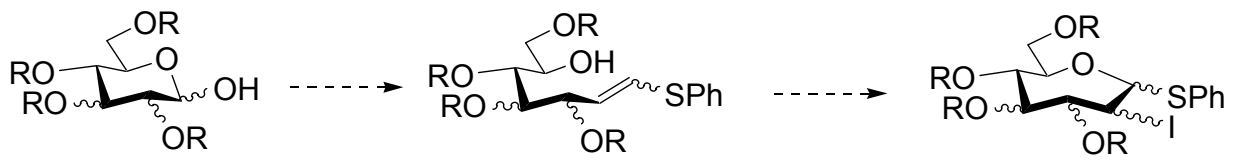

\section{Scheme 2}

In order to expand the scope of this strategy, we decided to explore the olefinationelectrophile-induced cyclization strategy as a route to 2-deoxy-2-iodo-septanosides (Scheme 2). ${ }^{8 b}$ There are few examples for the formation of oxepane rings by electrophile-induced cyclization and these are mainly related to the formation of lactones through a 7-exo cyclization mode. ${ }^{15}$ To the best of our knowledge, there is only one example of formation of oxepanes by iodine-induced cyclization of hydroxyl-enolethers through a 7 -endo cyclization. ${ }^{16}$ In this paper, we present our 
results on the synthesis of heptenyl thioethers derived from protected hexo-pyranoses and pentoses and the study of electrophile induced cyclization under various conditions.

\section{Results and Discussion}

As shown in previous studies, the Wittig-Horner conditions for the synthesis of sulfanyl-alkenes using phosphine oxide carbanions and $\mathrm{Li}$ bases were the most effective in terms of chemoselectivity, diastereoselectivity and yield. ${ }^{11}$ The selected olefination reagent diphenyl phenylsulfanylmethyl phosphineoxide was prepared by the Arbuzov reaction in 94\% yield starting from ethyl diphenyl phosphinite and chloromethyl phenyl sulfide. ${ }^{17}$

When the Wittig-Horner reaction was carried out starting from 2,3,4,6-tetra- $O$-benzyl-Dglucopyranose $\mathbf{1}$ and diphenyl phenylsulfanyl methylphosphine oxide, the expected product $\mathbf{2}$ was obtained in $63 \%$ yield as an inseparable mixture of diastereomers $(Z / E=1 / 8)$, as expected for semi-stabilized carbanions.

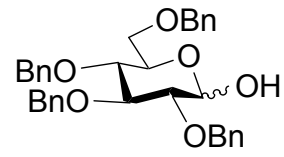

1
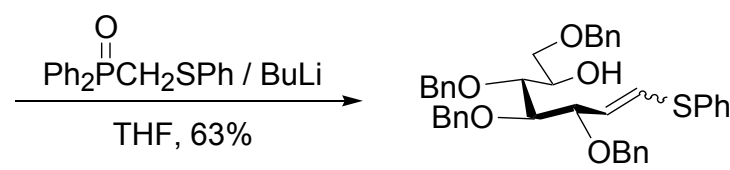

2

Scheme 3

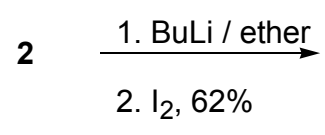

2. $\mathrm{I}_{2}, 62 \%$

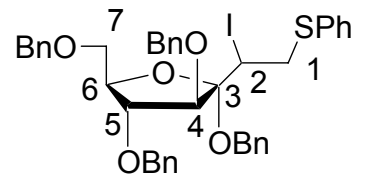

3

\section{Scheme 4}

Subsequently, the cyclization of the alkenylsulfanyl derivative $\mathbf{2}$ was studied. Initially, the standard conditions reported by Barlett, ${ }^{11,18}$ using iodine in acetronitrile in presence of $\mathrm{NaHCO}_{3}$, were tested. However, under these conditions only the starting material was recovered. Heating the mixture at $40{ }^{\circ} \mathrm{C}$ or using NIS as an electrophile was also ineffective.

Increasing the nucleophilicity of oxygen forming first the alkoxide was also tested. Treatment of compound 2 with $\mathrm{KH}$ and iodine in ether at $-78{ }^{\circ} \mathrm{C}$ did not provide the oxepane ring. However, reaction with $n$-Buli as a base afforded 3 in $62 \%$ yield (Scheme 4 ).

A similar behaviour had already been observed in the cyclization reaction of tri- $O$-benzylarabino derivative 4 in the presence of $\mathrm{KH}^{19}$, which led to the formation of oxetane $\mathbf{5}$ in $31 \%$ 
yield (Scheme 5). However, in the presence of a weak base, the cyclization product 6 was preferentially formed. ${ }^{11 \mathrm{a}}$

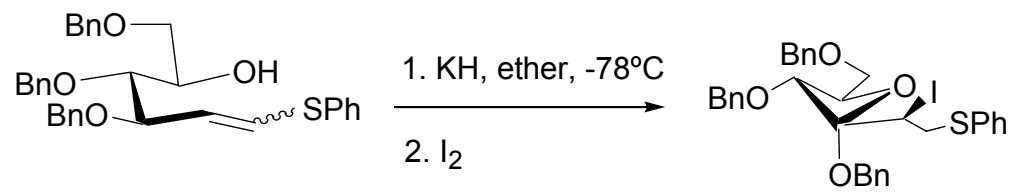

4

5

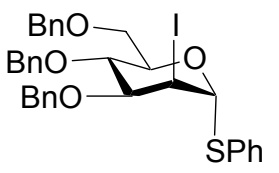

6

\section{Scheme 5}

These unexpected outcome takes place when cyclization is attempted using BuLi or $\mathrm{KH}$ as bases. Under strong basic conditions, the more nucleophilic alkoxide anion 7 was expected to be formed and eventually cyclize. However, and as already studied previously, ${ }^{11}$ the preferred conformations in the arabino and gluco derivatives do not favour cyclization because the allylic alkoxide group does not occupy an inside position with respect to the $\mathrm{C}=\mathrm{C}$ double bond, so that alternative reaction pathways are likely to take place. One of them could consist of a proton transfer to render an allylic anion $\mathbf{8}$ that reprotonates to give enol ether $\mathbf{9}$, which is considerable electron richer and hence more reactive towards cyclization than the starting enol thioether 7 (Scheme 6).

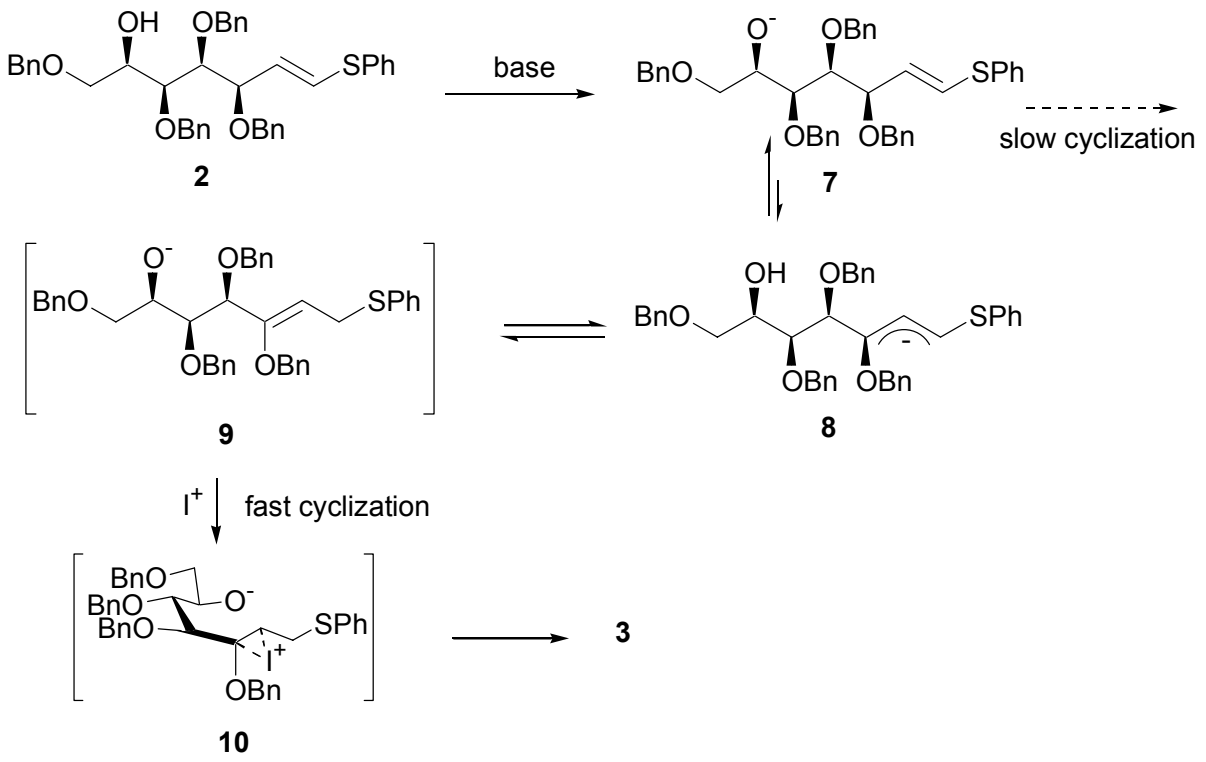

\section{Scheme 6}

Recently, the 7-membered ring glycal 12 was synthesized by a tungsten-induced cyclization starting from alkyne 11 (Scheme 7). ${ }^{7}$ The presence of an isopropylidene protecting group was necessary in order to favor the formation of the seven-membered ring. 


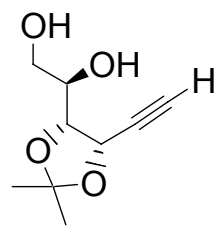

11
1) $15 \% \mathrm{~W}(\mathrm{CO})_{6}$

$\mathrm{Et}_{3} \mathrm{~N}, \mathrm{hv}$

2) $\mathrm{Ac}_{2} \mathrm{O}, \mathrm{Py}$

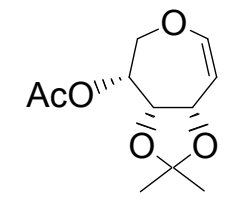

12

\section{Scheme 7}

In order to test how the presence of a dioxolane cycle in the starting material would favor the cyclization reaction, we prepared the sulfanyl alkene 14 by reaction of the ribose derivative 13 with $\mathrm{Ph}_{2} \mathrm{P}(\mathrm{O}) \mathrm{CH}_{2} \mathrm{SPh}$ in the presence of $\mathrm{BuLi}$. Then, the reaction of 14 with benzyl bromide afforded 15, which was subsequently treated with TBAF to give $\mathbf{1 6}$ (Scheme 8). Compound $17^{20}$ with hydroxyl groups at positions 5 and 6 was also prepared from 14 to study the competition between 6-endo and 7-endo cyclization.

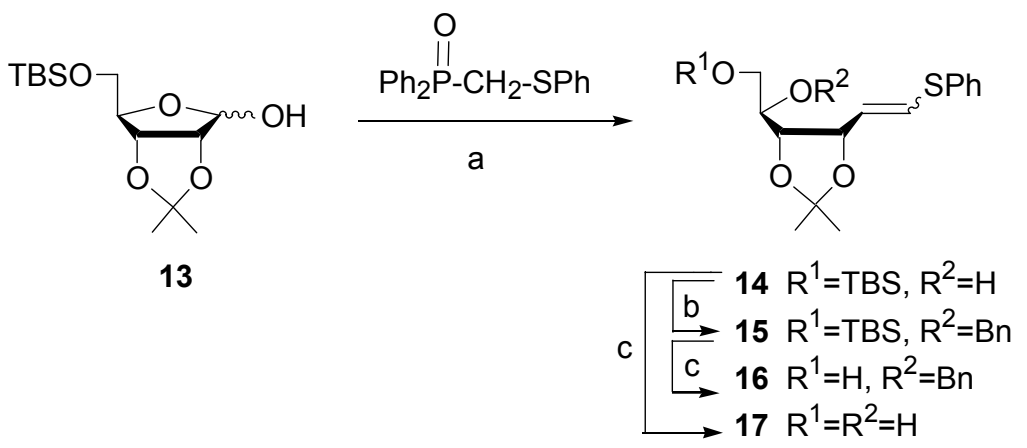

a) BuLi, THF, $68 \%$. b) NaH, BnBr, THF, 37\%. c) TBAF, THF, $96 \%$ for $16,98 \%$ for 17.

\section{Scheme 8}

When 14 was treated with NIS in basic media at low temperature, compound 19 was isolated in $46 \%$ yield, as a result of 6-endo cyclization to give 18 and concomitant loss of the silyl protecting group (Scheme 9). The stereochemical outcome of the reaction was similar to that previously observed for related compounds without isopropylidene protecting groups. ${ }^{11}$ Compound 19 was also exclusively obtained in similar yield starting from 17, which indicates that the 6-endo cyclization is preferred over the 7-endo.

When 16 was used as starting material, the reaction evolved much slower and required heating for a long period. After 24 hours at $35^{\circ} \mathrm{C}$, compound 20 was isolated in $12 \%$ yield (Scheme 9). The reaction was not complete and $40 \%$ of starting material was also recovered. The structure of $\mathbf{2 0}$ was determined according the following data: a) The signals of H1 and C1 which appear at chemical shifts, $5.56 \mathrm{ppm}$ and $93.0 \mathrm{ppm}$ respectively, characteristic of anomeric proton and carbon, and a $\mathrm{J}_{6 \mathrm{a}, 6 \mathrm{~b}}$ value of $13 \mathrm{~Hz}$, indicate that cyclization has taken place. $\mathrm{b}$ ) The 
presence of iodine at position 2 was confirmed by the correlation of $\mathrm{H} 2$ with a ${ }^{13} \mathrm{C}$ signal at 32 ppm (Table 1). c) From $\mathrm{J}_{1,2}$ and $\mathrm{J}_{2,3}$ values, an equatorial disposition for the substituents at these positions can be deduced. d) The presence of $\mathrm{H} 2$ on the botton face of the molecule was confirmed by the existence of NOE with the signal at $3.81 \mathrm{ppm}$ that corresponds to H6 axial.

It should be noted that the relative stereochemistry of iodine and the neighboring alkoxy group is trans, opposite to those observed for the cyclizations yielding pyranoses (Scheme 9), where the relative stereochemistry was always cis, as a result that cyclization takes place under the effect of the so-called alkoxy-inside effect. ${ }^{21}$ This effect establishes that in the more reactive conformer, the alkoxy chain occupies an inside conformation with respect to the double bond. The low reactivity observed can be due to the high substitution of the chain which limits the number of reactive conformations, and to the fact that cyclization in compound $\mathbf{1 6}$ takes place via the less reactive alkoxy-outside conformer.

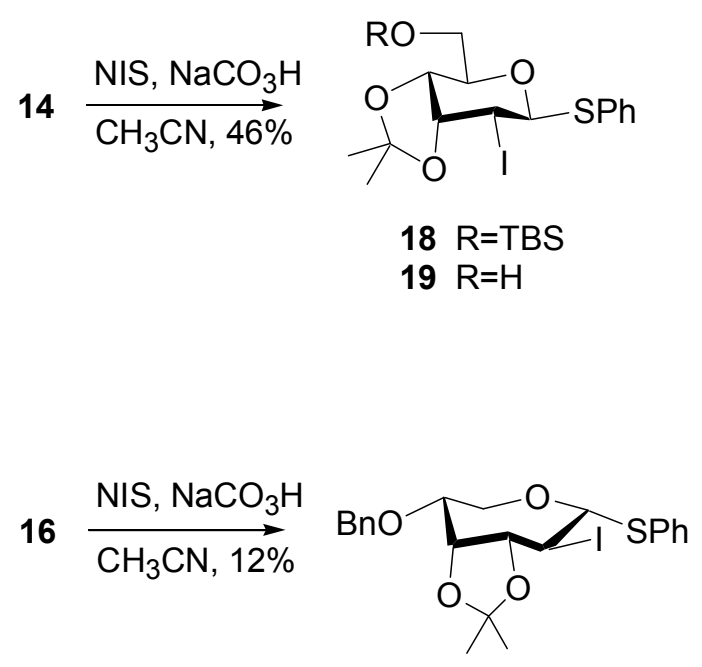

20

\section{Scheme 9}

In previous work, we prepared compound $\mathbf{2 2}$ by olefination of the lyxo derivative $\mathbf{2 1}$ (Scheme 10). ${ }^{11}$ Iodine-induced cyclization of 22 gave the 2-deoxy-2-iodo-1-thio-pyranoside 25 (Scheme 11). ${ }^{11}$ We had previously observed that benzyl ethers reacted in electrophile-induced cyclizations. $^{22}$ In order to avoid this possibility, compound 22 was protected as ethyl ether to give derivative 23, which was treated with TBAF to afford $\mathbf{2 4}$ (Scheme 10). When $\mathbf{2 4}$ was treated with $\mathrm{NIS} / \mathrm{NaCO}_{3} \mathrm{H}$, the reaction slowly evolved to give a mixture of compounds $\mathbf{2 6 \mathbf { a }}$ and $\mathbf{2 6 \mathbf { b }}$ in $36 \%$ yield (32\% of the starting material was also recovered) as an anomeric $\alpha / \beta$ mixture, resulting from a 7-endo cyclization followed by the hydrolysis of the anomeric phenylsulfanyl group (Scheme 12). This hydrolysis was already observed in other cases when the cyclization was slow, since the activation of the 1-thiophenyl group by NIS competes. ${ }^{11}$ More relevant spectroscopic features allowing the structural elucidation of $\mathbf{2 6 a}$, b are the following: a) ${ }^{13} \mathrm{C}$ 
chemical shifts at 96.9 and 98.1 ppm for $\mathrm{C} 1$ and at 35.4 and 32.5 for $\mathrm{C} 2$, for $\mathbf{2 6 a}$ and 26b respectively, together with the absence of aromatic carbons, confirms the presence of an hydroxyl group at $\mathrm{C} 1$ and an iodine at $\mathrm{C} 2$ (Table 1). b) The existence of acetalic carbons and the $\mathrm{J}_{6 \mathrm{a}, 6 \mathrm{~b}}$ value of $13 \mathrm{~Hz}$ confirms that compounds are cyclized. c) For compound 26a, the $\mathrm{J}_{2,3}$ value of $10.0 \mathrm{~Hz}$ indicates that these protons are in a trans-diaxial position, and the NOE cross peak observed between protons $\mathrm{H} 2$ and $\mathrm{H} 5$, confirms that iodine is on the $\alpha$-face. That suggests that for compound 26a the 7-endo cyclization has taken place under an alkoxy-outside control. Configuration of $\mathbf{2 6} \mathbf{b}$ could not be fully determined.

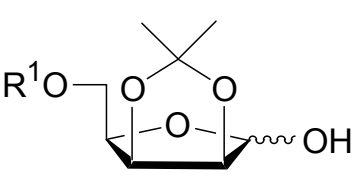

21

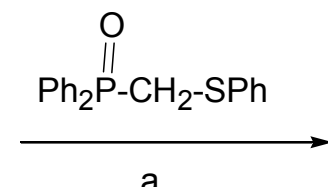

a
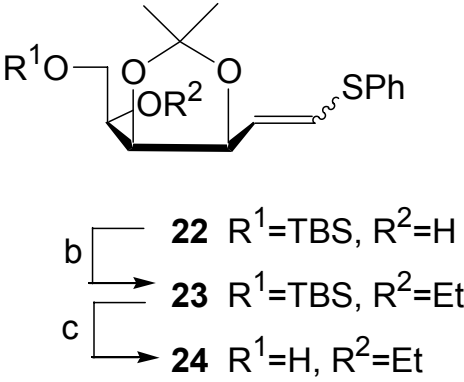

a) BuLi, THF, $68 \%$. b) NaH, EtBr,, THF, 37\%. c) TBAF, THF, $96 \%$

\section{Scheme 10}

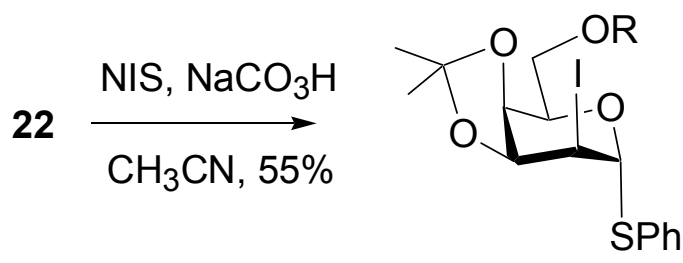

$25 \mathrm{R}=\mathrm{TBS}$

\section{Scheme 11}

$$
24 \underset{\mathrm{CH}_{3} \mathrm{CN}, 36 \%}{\stackrel{\mathrm{NIS}, \mathrm{NaCO}_{3} \mathrm{H}}{\longrightarrow}}
$$

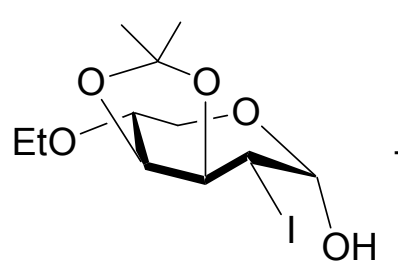

26a

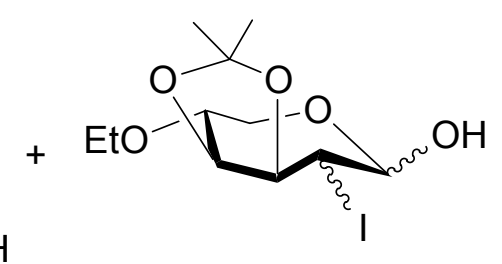

$1.4: 1$

26b

\section{Scheme 12}


Table 1. Selected ${ }^{1} \mathrm{H}$ - and ${ }^{13} \mathrm{C}-\mathrm{NMR}$ data of compounds 20, 26a and $\mathbf{2 6 \mathbf { b }}$ ( $\delta$ en ppm, J en Hz)

\begin{tabular}{cccccccccccccc}
\hline & $\mathrm{H} 1$ & $\mathrm{H} 2$ & $\mathrm{H} 3$ & $\mathrm{H} 4$ & $\mathrm{H} 5$ & $\mathrm{H} 6 \mathrm{a}$ & $\mathrm{H} 6 \mathrm{~b}$ & $\mathrm{~J}_{1,2}$ & $\mathrm{~J}_{2,3}$ & $\mathrm{~J}_{3,4}$ & $\mathrm{~J}_{4,5}$ & $\mathrm{~J}_{5,6 \mathrm{a}}$ & $\mathrm{J}_{5,6 \mathrm{~b}}$ \\
\hline $\mathbf{2 0}$ & 5.56 & 5.13 & 4.65 & 4.49 & 4.13 & 4.58 & 3.81 & 8.8 & 8.8 & 7.6 & 2.0 & 1.0 & 5.2 \\
$\mathbf{2 6 a}$ & 5.48 & 4.18 & 4.72 & 4.25 & 3.47 & 3.95 & 3.57 & 1.2 & 10.0 & 8.0 & 7.2 & 9.6 & 2.0 \\
$\mathbf{2 6 b}$ & 5.41 & 4.10 & 4.41 & 4.32 & 3.75 & 4.33 & 3.45 & 8.0 & nd & 7.2 & nd & nd & nd \\
\hline \multicolumn{2}{c}{} & & & & & & & & & & & \\
\hline & & $\mathrm{C} 1$ & & $\mathrm{C} 2$ & & $\mathrm{C} 3$ & & $\mathrm{C} 4$ & $\mathrm{C} 5$ & & $\mathrm{C} 6$ \\
\hline $\mathbf{2 0}$ & 93.0 & 32.0 & 80.1 & 76.9 & 77.8 & 63.5 \\
$\mathbf{2 6 a}$ & 96.9 & 35.4 & 76.5 & 80.4 & 78.8 & 60.7 \\
$\mathbf{2 6 b}$ & 98.1 & 32.5 & 77.0 & 78.5 & 78.1 & 62.0 \\
\hline
\end{tabular}

nd: not determined

\section{Conclusions}

Septanosides 20 and 26 were obtained in low to moderate yields from pentoses through a twostep procedure. A Wittig-Horner olefination of pentoses 13 and 21 gave the phenylsulphanyl derivatives 14 and 22, further protection and deprotection gave compounds 16 and 24, and NISinduced 7-endo cyclization afforded compounds 20 and 26. 7-Endo cyclization took place, preferably under alkoxy-outside control, when an isopropylidene protecting group was present in the starting alkene. This was the first example of 7-endo iodine-induced-cyclization to give highly substituted oxepanes. In the absence of an isopropylidene group the cyclization did not take place and when more basic reaction conditions were used, in order to force the cyclization, the reaction evolved in a different way to yield compound $\mathbf{3}$.

\section{Experimental Section}

General Procedures. Optical rotations were measured at room temperature in $10 \mathrm{~cm}$ cells in a Perkin-Elmer 241 polarimeter. ${ }^{1} \mathrm{H},{ }^{13} \mathrm{C}$ and ${ }^{31} \mathrm{P}$ NMR spectra were recorded using a Varian Gemini $300 \mathrm{MHz}$ and $400 \mathrm{MHz}$ apparatus, with $\mathrm{CDCl}_{3}$ as solvent, $\mathrm{Me}_{4} \mathrm{Si}$ as an internal reference, and $\mathrm{H}_{3} \mathrm{PO}_{4}\left({ }^{31} \mathrm{P}\right)$ as external standard, unless specified otherwise. Elemental analyses were performed using a Carlo-Erba Microanalyzer. Flash column chromatography was performed using silica gel 60 A CC (230-400 mesh). Radial chromatography was performed on 1, 2 or $4 \mathrm{~mm}$ plates of Kieselgel $60 \mathrm{PF}_{254}$ silica gel, depending on the amount of product. Medium-pressure chromatography (MPLC) was performed using silica gel 60 A CC (6-35 $\mu \mathrm{m})$. Solvents were purified using standard procedures. 
(Z/E)-3,4,5,7-Tetra-O-benzyl-1,2-dideoxy-1-phenylsulfanyl-D-gluco-hept-1-enitol

(2).

Diphenyl phenylsulfanyl methylphosphine oxide $(1.3 \mathrm{~g}, 4.0 \mathrm{mmol})$ was dissolved in anhydrous THF (27 ml, 0.15 M). $n$-BuLi (2.6 ml, $4.2 \mathrm{mmol}, 1.6 \mathrm{M}$ in hexane) was slowly added at $-78{ }^{\circ} \mathrm{C}$, stirred under an argon atmosphere until the intensive orange colour was formed. The reaction mixture was stirred for an hour at this temperature, and the THF solution of the 2,3,4,6-tetra- $O$ benzyl-D-glucopyranose $(2.0 \mathrm{ml}, 0.5 \mathrm{M})$ was added. The evolution of the reaction was monitored by TLC analysis with general sugar dye. The reaction did not evolve at $-78{ }^{\circ} \mathrm{C}$ and the solution was warmed up to room temperature. After full conversion $(24 \mathrm{~h})$ and work up, the product was purified by flash chromatography (hexane:ethyl acetate $=3: 1$ ) to obtain the vinyl phenylsulfanyl compound (406 mg, $0.6278 \mathrm{mmol}, 63 \%$ ) as an inseparable mixture of $Z / E=1 / 8$ as an oil.

NMR spectral data were extracted from the diastereoisomeric mixture. $\boldsymbol{E}-\mathbf{2}$ : $\mathrm{R}_{\mathrm{f}}$ (hexane:ethyl acetate=2:1): 0.7. ${ }^{1} \mathrm{H} \mathrm{NMR}\left(\mathrm{CDCl}_{3}, 400 \mathrm{MHz}\right) \delta$ in ppm: 7.52-7.16 $(25 \mathrm{H}, m, \mathrm{Ar}), 6.30(1 \mathrm{H}, d, \mathrm{~J}=$ $15.2 \mathrm{~Hz}, \mathrm{H}-1), 5.70(1 \mathrm{H}, d d, \mathrm{~J}=15.2,8.0 \mathrm{~Hz}, \mathrm{H}-2), 4.80\left(1 \mathrm{H}, d, \mathrm{~J}=11.2 \mathrm{~Hz}, \mathrm{CH}_{2} \mathrm{Ph}\right), 4.71(1 \mathrm{H}$, $\left.d, \mathrm{~J}=11.2 \mathrm{~Hz}, \mathrm{CH}_{2} \mathrm{Ph}\right), 4.67\left(1 \mathrm{H}, d, \mathrm{~J}=11.2 \mathrm{~Hz}, \mathrm{CH}_{2} \mathrm{Ph}\right), 4.62\left(1 \mathrm{H}, d, \mathrm{~J}=11.2 \mathrm{~Hz}, \mathrm{CH}_{2} \mathrm{Ph}\right)$, $4.64\left(\mathrm{H} 1, \mathrm{~J}=11.2 \mathrm{~Hz}, \mathrm{CH}_{2} \mathrm{Ph}\right), 4.50\left(2 \mathrm{H}, s, \mathrm{CH}_{2} \mathrm{Ph}\right), 4.48\left(1 \mathrm{H}, d, \mathrm{~J}=11.2 \mathrm{~Hz}, \mathrm{CH}_{2} \mathrm{Ph}\right), 4.24(1 \mathrm{H}$, $d d, \mathrm{~J}=8.0,5.6 \mathrm{~Hz}, \mathrm{H}-3), 4.01(1 \mathrm{H}, m, \mathrm{H}-5), 3.72(2 \mathrm{H}, m, \mathrm{H}-6), 3.60$ (1H, $s, \mathrm{H}-7 \mathrm{a}), 3.58$ (1H, $d, \mathrm{~J}$ $=2.0 \mathrm{~Hz}, \mathrm{H}-7 \mathrm{~b}), 2.82(1 \mathrm{H}, d, \mathrm{~J}=6.0 \mathrm{~Hz}, \mathrm{OH}) .{ }^{13} \mathrm{C} \mathrm{NMR}\left(\mathrm{CDCl}_{3}, 100.6 \mathrm{MHz}\right) \delta$ in ppm: 138.5 , 138.3, 138.2, 138.1, 134.4 (C, Ar), 130.6-127.4 (CH, Ar), 128.6 (CH, C-1), 128.4 (CH, C-2), $81.6(\mathrm{CH}, \mathrm{C}-4), 81.0(\mathrm{CH}, \mathrm{C}-3), 78.6(\mathrm{CH}, \mathrm{C}-6), 75.0,73.5,73.34\left(\mathrm{CH}_{2} \mathrm{Ph}\right), 71.2\left(\mathrm{CH}_{2}, \mathrm{C}-7\right)$, $71.0\left(\mathrm{CH}_{2} \mathrm{Ph}\right), 70.5(\mathrm{CH}, \mathrm{C}-5)$.

Z-2: $\mathrm{R}_{\mathrm{f}}$ (hexane:ethyl acetate=2:1): 0.6. ${ }^{1} \mathrm{H} \mathrm{NMR}\left(\mathrm{CDCl}_{3}, 400 \mathrm{MHz}\right) \delta$ in ppm: 7.35-7.18 $(25 \mathrm{H}$, $m, \operatorname{Ar}), 6.50(1 \mathrm{H}, d, \mathrm{~J}=9.2 \mathrm{~Hz}, \mathrm{H}-1), 5.89(1 \mathrm{H}, d d, \mathrm{~J}=9.2,8.8 \mathrm{~Hz}, \mathrm{H}-2), 4.80-4.40(8 \mathrm{H}, d$, $\left.\mathrm{CH}_{2} \mathrm{Ph}\right), 4.24(1 \mathrm{H}, m, \mathrm{H}-3), 4.0(1 \mathrm{H}, m, \mathrm{H}-5), 3.82(2 \mathrm{H}, m, \mathrm{H}-4, \mathrm{H}-6), 3.60$ (1H, $\left.s, \mathrm{H}-7 \mathrm{a}\right), 3.58$ $(1 \mathrm{H}, d, \mathrm{~J}=2.0 \mathrm{~Hz}, \mathrm{H}-7 \mathrm{~b}), 2.87(1 \mathrm{H}, d, \mathrm{~J}=5.2 \mathrm{~Hz}, \mathrm{OH})$.

2,3,4-Tri-benzyloxy-5-benzyloxymethyl-2-(1-iodo-2-phenylsulfanyl-ethyl)-tetrahydro-furan

(3). To a solution of $2(55 \mathrm{mg}, 0.085 \mathrm{mmol})$ in $1.0 \mathrm{ml}(0.085 \mathrm{M})$ of anhydrous diethyl ether, 53 $\mu \mathrm{l}$ of $n$-BuLi $\left(0.085 \mathrm{mmol}, 1.6 \mathrm{M}\right.$ in hexane) were added at $-78{ }^{\circ} \mathrm{C}$. The mixture was stirred for one hour at this temperature under an argon atmosphere. Subsequently, a solution of $\mathrm{I}_{2}(65 \mathrm{mg}$, $0.225 \mathrm{mmol})$ in $2.0 \mathrm{ml}(0.43 \mathrm{M})$ of diethyl ether was added. TLC analysis showed the completion of the reaction after $5 \mathrm{~min}$. The reaction was quenched with $\mathrm{Na}_{2} \mathrm{~S}_{2} \mathrm{O}_{3}$, and the aqueous layer was extracted three times with ethyl acetate. The combined organic layers were washed with water, dried on anhydrous $\mathrm{MgSO}_{4}$, and concentrated under vacuum. After purification by radial chromatography methods (hexane $\rightarrow$ hexane:ethyl acetate $=1: 2), 3$ (41 mg, $0.053 \mathrm{mmol}, 62 \%$ ) was obtained as an oil.

$\mathrm{R}_{\mathrm{f}}$ (hexane:ethyl acetate=2:1): 0.67. $\left.[\alpha]_{\mathrm{D}}{ }^{25}-21.5(c) 0.40, \mathrm{CH}_{2} \mathrm{Cl}_{2}\right) .{ }^{1} \mathrm{H}-\mathrm{NMR}{ }^{23}\left(\mathrm{CDCl}_{3}, 400\right.$ $\mathrm{MHz}) \delta$ in ppm: 7.25-7.13 $(25 \mathrm{H}, m, \mathrm{Ar}), 4.81\left(1 \mathrm{H}, d, \mathrm{~J}=11.2 \mathrm{~Hz}, \mathrm{CH}_{2} \mathrm{Ph}\right), 4.65(1 \mathrm{H}, d, \mathrm{~J}=11.2$ $\left.\mathrm{Hz}, \mathrm{CH}_{2} \mathrm{Ph}\right), 4.61\left(1 \mathrm{H}, d, \mathrm{~J}=11.2 \mathrm{~Hz}, \mathrm{CH}_{2} \mathrm{Ph}\right), 4.58\left(1 \mathrm{H}, d, \mathrm{~J}=11.2 \mathrm{~Hz}, \mathrm{CH}_{2} \mathrm{Ph}\right), 4.56(1 \mathrm{H}, d, \mathrm{~J}$ $=6.4 \mathrm{~Hz}, \mathrm{H}-4), 4.49\left(1 \mathrm{H}, d, \mathrm{~J}=11.2 \mathrm{~Hz}, \mathrm{CH}_{2} \mathrm{Ph}\right), 4.46\left(1 \mathrm{H}, d, \mathrm{~J}=11.2 \mathrm{~Hz}, \mathrm{CH}_{2} \mathrm{Ph}\right), 4.44(1 \mathrm{H}, d$, $\left.\mathrm{J}=11.2 \mathrm{~Hz}, \mathrm{CH}_{2} \mathrm{Ph}\right), 4.38\left(1 \mathrm{H}, d, \mathrm{~J}=11.6 \mathrm{~Hz}, \mathrm{CH}_{2} \mathrm{Ph}\right), 4.30(1 \mathrm{H}, d d, \mathrm{~J}=7.2,6.4 \mathrm{~Hz}, \mathrm{H}-5), 4.18$ $(1 \mathrm{H}, d d, \mathrm{~J}=10.8,2.8 \mathrm{~Hz}, \mathrm{H}-2), 4.12(1 \mathrm{H}, m, \mathrm{H}-6), 3.86\left(1 \mathrm{H}, d d, \mathrm{~J}=14.8,2.8 \mathrm{~Hz}, \mathrm{CH}_{2}, \mathrm{H}-1 \mathrm{a}\right)$, 
$3.69(1 \mathrm{H}, d d, \mathrm{~J}=10.8,2.8 \mathrm{~Hz}, \mathrm{H}-7 \mathrm{a}), 3.53(1 \mathrm{H}, d d, \mathrm{~J}=10.8,3.6 \mathrm{~Hz}, \mathrm{H}-7 \mathrm{~b}) 3.22(1 \mathrm{H}, d d, \mathrm{~J}=$ $\left.14.8,10.8 \mathrm{~Hz}, \mathrm{CH}_{2}, \mathrm{H}-1 \mathrm{~b}\right) .{ }^{13} \mathrm{C}-\mathrm{NMR}{ }^{22}\left(\mathrm{CDCl}_{3}, 100.6 \mathrm{MHz}\right) \delta$ in ppm: 139.0, 138.4, 138.4, 138.1, 136.0 (C, Ar), 129.6-125.3 (CH, Ar), 105.3 (C, C-3), 87.2 (CH, C-4), 82.9 (CH, C-5), 80.6 (CH, C-6), 73.8, 73.1, $72.9\left(\mathrm{CH}_{2} \mathrm{Ph}\right), 69.5\left(\mathrm{CH}_{2}, \mathrm{C}-7\right), 65.8\left(\mathrm{CH}_{2} \mathrm{Ph}\right), 41.9(\mathrm{CH}, \mathrm{C}-2), 40.1$ $\left(\mathrm{CH}_{2}, \mathrm{C}-1\right)$. HRMS (TOF MS ES+): calcd for $\mathrm{C}_{41} \mathrm{H}_{41} \mathrm{O}_{5} \mathrm{NaSI} \quad(\mathrm{MNa}+)$ 795.1617; found, 795.1600 .

\section{(Z/E)-6-O-tert-Butyldimethylsilyl-1,2-dideoxy-3,4-O-isopropylidene-1-phenylsulfanyl-D-}

ribo-hex-1-enitol (14). Diphenyl phenylsulfanylmethylphosphine oxide (4.26 g, $13.1 \mathrm{mmol})$ was dissolved in anhydrous THF (13 ml, 0.25 M). $n$-BuLi ( $8.6 \mathrm{ml}, 13.8 \mathrm{mmol}, 1.6 \mathrm{M}$ in hexane) was added slowly at $-78{ }^{\circ} \mathrm{C}$ and the solution was stirred under an argon atmosphere until the intensive orange colour occurred. The reaction mixture was stirred for an hour at this temperature, then the solution of $\mathbf{1 3}(1.0 \mathrm{~g}, 3.3 \mathrm{mmol})$ in anhydrous THF $(2.0 \mathrm{ml}, 0.5 \mathrm{M})$ was added. After full conversion $(24 \mathrm{~h})$ and work up, the product was purified by flash chromatography (hexane: ethyl acetate $=6: 1$ ) to obtain compound $\mathbf{1 4}$ as an inseparable mixture of $Z / E=1 / 11$ (920 mg, $2.24 \mathrm{mmol}, 68 \%)$ as an oil.

NMR spectral data were extracted from the diastereoisomeric mixture. $\boldsymbol{E}-14: \mathrm{R}_{\mathrm{f}}$ (hexane:ethyl acetate=6:1): 0.62. ${ }^{1} \mathrm{H}-\mathrm{NMR}^{23}\left(\mathrm{CDCl}_{3}, 400 \mathrm{MHz}\right) \delta$ in ppm: 7.51-7.20 (5H, $\left.m, \mathrm{Ar}\right), 6.50(1 \mathrm{H}, d, \mathrm{~J}$ $=15.2 \mathrm{~Hz}, \mathrm{H}-1), 6.00(1 \mathrm{H}, d d, \mathrm{~J}=15.2,6.4 \mathrm{~Hz}, \mathrm{H}-2), 4.77(1 \mathrm{H}, d d, \mathrm{~J}=6.4,6.0 \mathrm{~Hz}, \mathrm{H}-3), 4.05$ $(1 \mathrm{H}, d d, \mathrm{~J}=9.2,6.0 \mathrm{~Hz}, \mathrm{H}-4), 3.81(1 \mathrm{H}, d d, \mathrm{~J}=9.6,3.2 \mathrm{~Hz}, \mathrm{H}-6 \mathrm{a}), 3.68(\mathrm{H}, d d, \mathrm{~J}=9.6,5.2 \mathrm{~Hz}$, $\mathrm{H}-6 \mathrm{~b}), 3.64(1 \mathrm{H}, m, \mathrm{H}-5), 2.50(1 \mathrm{H}, d, \mathrm{~J}=6.0 \mathrm{~Hz}, \mathrm{OH}), 1.46\left(3 \mathrm{H}, s, \mathrm{CH}_{3}\right), 1.35\left(3 \mathrm{H}, s, \mathrm{CH}_{3}\right)$, $0.92\left(9 \mathrm{H}, s, \mathrm{CH}_{3}\right), 0.12\left(6 \mathrm{H}, s, \mathrm{CH}_{3}\right) .{ }^{13} \mathrm{C} \mathrm{NMR}\left(\mathrm{CDCl}_{3}, 100.6 \mathrm{MHz}\right) \delta$ in ppm: 139.2, $(\mathrm{C}, \mathrm{Ar})$, 130.0-127.0 (CH, Ar), 127.3 (CH, C-1), 126.7 (CH, C-2), $109.5(\mathrm{C}), 78.5(\mathrm{CH}, \mathrm{C}-3), 76.9(\mathrm{CH}$, C-4), $69.0\left(\mathrm{CH}_{2}, \mathrm{C}-6\right), 64.6(\mathrm{CH}, \mathrm{C}-5), 28.1\left(\mathrm{CH}_{3}\right), 26.1\left(\mathrm{CH}_{3}\right), 25.6\left(\mathrm{CH}_{3}\right)$.

\section{$(Z / E)$-5-O-benzyl-6-O-tert-butyldimethylsilyl-1,2-dideoxy-3,4-O-isopropylidene-1-}

phenylsulfanyl-D-ribo-hex-1-enitol (15). To a suspension of sodium hydride ( $84 \mathrm{mg}, 2.1 \mathrm{mmol})$ in THF, compound $14(820 \mathrm{mg}, 2.0 \mathrm{mmol})$ dissolved in freshly distilled THF $(8.0 \mathrm{ml}, 0.25 \mathrm{M})$ was added at room temperature. The reaction mixture was further stirred for an hour and benzyl bromide $(250 \mu \mathrm{l}, 2.1 \mathrm{mmol})$ was slowly added. The reaction mixture was stirred overnight, and the evolution of the reaction was followed by TLC analysis. The reaction was quenched by saturated ammonium chloride solution. The aqueous layer was extracted with ethyl acetate $(3 \times 20$ $\mathrm{ml})$, the combined organic layers were washed with water $(2 \times 20 \mathrm{ml})$, with brine $(1 \times 20 \mathrm{ml})$ and dried on $\mathrm{MgSO}_{4}$, filtered and concentrated under vacuum. The resulting mixture was purified by chromatography (hexane $\rightarrow$ hexane:ethyl acetate $=3: 1$ ) to obtain compound $\mathbf{1 5}$ as a light yellow oil (361 mg, $0.74 \mathrm{mmol}, 37 \%$ ) as an inseparable mixture of $Z / E=1 / 11$.

NMR spectral data were extracted from the diastereoisomeric mixture. $\boldsymbol{E}-15: \mathrm{R}_{\mathrm{f}}$ (hexane:ethyl acetate=8:1): 0.51. ${ }^{1} \mathrm{H}-\mathrm{NMR}^{23}\left(\mathrm{CDCl}_{3}, 400 \mathrm{MHz}\right) \delta$ in ppm: 7.40-7.22 (10H, $\left.m, \mathrm{Ar}\right), 6.52(1 \mathrm{H}, d$, $\mathrm{J}=15.6 \mathrm{~Hz}, \mathrm{H}-1), 5.86(1 \mathrm{H}, d d, \mathrm{~J}=15.6,6.4 \mathrm{~Hz}, \mathrm{H}-2), 4.77\left(1 \mathrm{H}, d, \mathrm{~J}=11.2 \mathrm{~Hz} \mathrm{CH}_{2} \mathrm{Ph}\right), 4.74$ $(1 \mathrm{H}, d d, \mathrm{~J}=6.4,5.6 \mathrm{~Hz}, \mathrm{H}-3), 4.40\left(1 \mathrm{H}, d, \mathrm{~J}=11.2 \mathrm{~Hz} \mathrm{CH}_{2} \mathrm{Ph}\right), 4.26(1 \mathrm{H}, d d, \mathrm{~J}=8.8,5.6 \mathrm{~Hz}, \mathrm{H}-$ 4), $3.86\left(1 \mathrm{H}, d d, \mathrm{~J}=10.0,2.0 \mathrm{~Hz}, \mathrm{CH}_{2}, \mathrm{H}-6 \mathrm{a}\right), 3.68\left(\mathrm{H}, d d, \mathrm{~J}=10.0,5.2 \mathrm{~Hz}, \mathrm{CH}_{2}, \mathrm{H}-6 \mathrm{~b}\right), 3.61$ $(1 \mathrm{H}, m, \mathrm{H}-5), 1.45\left(3 \mathrm{H}, s, \mathrm{CH}_{3}\right), 1.35\left(3 \mathrm{H}, s, \mathrm{CH}_{3}\right), 0.92\left(9 \mathrm{H}, s, \mathrm{CH}_{3}\right), 0.12\left(6 \mathrm{H}, s, \mathrm{CH}_{3}\right) .{ }^{13} \mathrm{C}$ 
NMR $\left(\mathrm{CDCl}_{3}, 100.6 \mathrm{MHz}\right) \delta$ in ppm: 139.0, 134.5 (C, Ar), 130.0-127.0 (CH, Ar), $127.3(\mathrm{CH}, \mathrm{C}-$ 1), 126.7 (CH, C-2), $109.5(\mathrm{C}), 78.5$ (CH, C-3), $76.9(\mathrm{CH}, \mathrm{C}-4), 72.3\left(\mathrm{CH}_{2}, \mathrm{CH}_{2} \mathrm{Ph}\right), 69.8\left(\mathrm{CH}_{2}\right.$, C-6), $64.6(\mathrm{CH}, \mathrm{C}-5), 28.1\left(\mathrm{CH}_{3}\right), 26.1\left(\mathrm{CH}_{3}\right), 25.6\left(\mathrm{CH}_{3}\right)$.

\section{(Z/E)-5-O-Benzyl-1,2-dideoxy-3,4-O-isopropylidene-1-phenylsulfanyl-D-ribo-hex-1-enitol}

(16). Compound $15(361 \mathrm{mg}, 0.74 \mathrm{mmol})$ was dissolved in THF $(3.0 \mathrm{ml})$ and tetrabutylammonium fluoride $(275 \mathrm{mg}, 0.78 \mathrm{mmol}$ ) was added. The reaction mixture was stirred at room temperature and the reaction was controlled by TLC analysis. After an hour, the reaction was quenched with a saturated sodium carbonate solution. The aqueous layer was extracted with ethyl acetate $(3 \times 20 \mathrm{ml})$, and the combined organic layers were washed with water $(2 \times 20 \mathrm{ml})$, with brine $(1 \times 20 \mathrm{ml})$ dried on $\mathrm{MgSO}_{4}$, filtered and concentrated under vacuum. The mixture was separated by chromatography (hexane $\rightarrow$ hexane:ethyl acetate $=1: 1$ ) and compound $\mathbf{1 6}$ was obtained as a light yellow oil $(276 \mathrm{mg}, 0.7136 \mathrm{mmol}, 96 \%)$ as an inseparable mixture of $Z / E=$ $1 / 11$.

NMR spectral data were extracted from the diastereoisomeric mixture. $\boldsymbol{E}-\mathbf{1 6}$ : $\mathrm{R}_{\mathrm{f}}$ (hexane:ethyl acetate=4:1): 0.46. ${ }^{1} \mathrm{H}-\mathrm{NMR}^{23}\left(\mathrm{CDCl}_{3}, 400 \mathrm{MHz}\right) \delta$ in ppm: 7.43-7.20 $(10 \mathrm{H}, \mathrm{m}, \mathrm{Ar}), 6.50(1 \mathrm{H}, \mathrm{d}$, $\mathrm{J}=15.2 \mathrm{~Hz}, \mathrm{H}-1), 5.88(1 \mathrm{H}, \mathrm{dd}, \mathrm{J}=15.2,6.8 \mathrm{~Hz}, \mathrm{H}-2), 4.77\left(1 \mathrm{H}, \mathrm{d}, \mathrm{J}=10.8 \mathrm{~Hz}, \mathrm{CH}_{2} \mathrm{Ph}\right), 4.76$ $(1 \mathrm{H}, \mathrm{dd}, \mathrm{J}=6.8,6.0, \mathrm{H}-3), 4.40\left(2 \mathrm{H}, \mathrm{d}, \mathrm{J}=10.8 \mathrm{~Hz}, \mathrm{CH}_{2} \mathrm{Ph}\right), 4.26,(1 \mathrm{H}, \mathrm{dd}, \mathrm{J}=8.8,6.0 \mathrm{~Hz}, \mathrm{H}-4)$, $3.86(1 \mathrm{H}, \mathrm{d}, \mathrm{J}=10.4 \mathrm{~Hz}, \mathrm{H}-6 \mathrm{a}), 3.66$ (1H, dd, J = 10.4, $5.2 \mathrm{~Hz}, \mathrm{H}-6 \mathrm{~b}), 3.65$ (1H, m, H-5), 1.45 $\left(3 \mathrm{H}, \mathrm{s}, \mathrm{CH}_{3}\right), 1.35\left(3 \mathrm{H}, \mathrm{s}, \mathrm{CH}_{3}\right) \cdot{ }^{13} \mathrm{C} \mathrm{NMR}\left(\mathrm{CDCl}_{3}, 100.6 \mathrm{MHz}\right) \delta$ in ppm: $138.4,134.79(\mathrm{C}, \mathrm{Ar})$, 130.3-126.5 (CH, Ar), $127.3(\mathrm{CH}, \mathrm{C}-1), 126.7$ (CH, C-2), $108.9(\mathrm{C}), 78.4(\mathrm{CH}, \mathrm{C}-3), 77.3(\mathrm{CH}$, C-5), $76.9(\mathrm{CH}, \mathrm{C}-4), 72.3\left(\mathrm{CH}_{2}, \mathrm{CH}_{2} \mathrm{Ph}\right), 70.6\left(\mathrm{CH}_{2}, \mathrm{C}-6\right), 29.8\left(\mathrm{CH}_{3}\right), 28.9\left(\mathrm{CH}_{3}\right)$.

(Z/E)-1,2-Dideoxy-3,4-O-isopropylidene-1-phenylsulfanyl-D-ribo-hex-1-enitol

(17).

Compound 14 (410.1 mg, $1 \mathrm{mmol})$ was dissolved in THF $(4.0 \mathrm{ml}, 0.25 \mathrm{M})$ and tetrabutyl ammonium fluoride $(331.3 \mathrm{mg}, 1.05 \mathrm{mmol}$ ) was added. The reaction mixture was stirred at room temperature and the reaction was controlled by TLC analysis. After an hour, the reaction was quenched with a saturated sodium carbonate solution. The aqueous layer was extracted with ethyl acetate $(3 \times 20 \mathrm{ml})$, the combined organic layers were washed with water $(2 \times 20 \mathrm{ml})$, with brine $(1 \times 20 \mathrm{ml})$, dried on $\mathrm{MgSO}_{4}$, filtered and concentrated under vacuum. The mixture was separated by chromatography (hexane $\rightarrow$ hexane:ethyl acetate $=1: 1$ ) and compound $\mathbf{1 7}$ was obtained as a light yellow oil (244 $\mathrm{mg}, 0.823 \mathrm{mmol}, 98 \%)$ as an inseparable mixture of $Z / E=$ $1 / 11$.

NMR spectral data were extracted from the diastereoisomeric mixture. $\boldsymbol{E}-17$ : $\mathrm{R}_{\mathrm{f}}$ (hexane:ethyl acetate=6:1): 0.62. ${ }^{1} \mathrm{H}-\mathrm{NMR}^{23}\left(\mathrm{CDCl}_{3}, 400 \mathrm{MHz}\right) \delta$ in ppm: 7.97-7.23 $(5 \mathrm{H}, m, \mathrm{Ar}), 6.58(1 \mathrm{H}, d$, $\mathrm{J}=14.8 \mathrm{~Hz}, \mathrm{H}-1), 5.90(1 \mathrm{H}, d d, \mathrm{~J}=14.8,6.8 \mathrm{~Hz}, \mathrm{H}-2), 4.78(1 \mathrm{H}, d d, \mathrm{~J}=6.8,6.0 \mathrm{~Hz}, \mathrm{H}-3), 4.09$ $(1 \mathrm{H}, d d, \mathrm{~J}=8.8,6.0 \mathrm{~Hz}, \mathrm{H}-4), 3.86(1 \mathrm{H}, d, \mathrm{~J}=10.4 \mathrm{~Hz}, \mathrm{H}-6 \mathrm{a}), 3.68(1 \mathrm{H}, m, \mathrm{H}-5), 3.66(1 \mathrm{H}, d d, \mathrm{~J}$

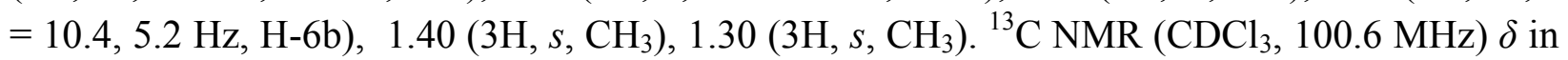
ppm: 134.5 (C, Ar), 130.4-127.3 (CH, Ar), 128.0 (CH, C-1), $126.9(\mathrm{CH}, \mathrm{C}-2), 109.2$ C), 78.2 $(\mathrm{CH}, \mathrm{C}-3), 76.8(\mathrm{CH}, \mathrm{C}-5), 74.84(\mathrm{CH}, \mathrm{C}-4), 70.0\left(\mathrm{CH}_{2}, \mathrm{C}-6\right), 27.8\left(\mathrm{CH}_{3}\right), 25.4\left(\mathrm{CH}_{3}\right)$.

Phenyl 2-deoxy-2-iodo-3,4-O-isopropylidene-1-thio- $\beta$-D-allo-pyranoside (19). Compound 14 (264 mg, $0.503 \mathrm{mmol}$ ) was dissolved in acetonitrile $(9.4 \mathrm{ml}, 0.05 \mathrm{M})$ and the solution was cooled 
to $-30{ }^{\circ} \mathrm{C}$. Sodium bicarbonate $(59 \mathrm{mg}, 0.70 \mathrm{mmol})$ and NIS $(158.6 \mathrm{mg}, 0.70 \mathrm{mmol})$ were then added. The reaction was controlled by TLC. After half an hour, full conversion was observed and the reaction was stopped by the addition of a saturated solution of sodium thiosulphate. The aqueous layer was extracted with ethyl acetate $(3 \times 20 \mathrm{ml})$, the combined organic layers were washed with water $(2 \times 20 \mathrm{ml})$, with brine $(1 \times 20 \mathrm{ml})$ dried on $\mathrm{MgSO}_{4}$, filtered and concentrated under vacuum. The crude reaction mixture was purified by chromatography (hexane $\rightarrow$ hexane:ethyl acetate $=1: 1)$ and compound 19 was obtained as a light yellow oil (101 mg, 0.239 mmol, 47\%).

$\mathrm{R}_{\mathrm{f}}$ (hexane: ethyl acetate $\left.=4: 1\right): 0.62 .{ }^{1} \mathrm{H}-\mathrm{NMR}^{23}\left(\mathrm{CDCl}_{3}, 400 \mathrm{MHz}\right) \delta$ in ppm 7.51-7.26 (5H, $\left.\mathrm{Ar}\right)$, $5.60(1 \mathrm{H}, d, \mathrm{~J}=3.6 \mathrm{~Hz}, \mathrm{H}-1), 4.64-4.57(2 \mathrm{H}, m, \mathrm{H}-2), 4.43(1 \mathrm{H}, d d, \mathrm{~J}=9.2,5.6 \mathrm{~Hz}, \mathrm{H}-3), 4.43$ $(1 \mathrm{H}, d d, \mathrm{~J}=9.2,5.6 \mathrm{~Hz}, \mathrm{H}-4), 4.29(1 \mathrm{H}, m, \mathrm{H}-5), 3.93(1 \mathrm{H}, d d, \mathrm{~J}=12.0,2.8 \mathrm{~Hz}, \mathrm{H}-6 \mathrm{a}), 3.79(1 \mathrm{H}$, $d d, \mathrm{~J}=12.0,5.2 \mathrm{~Hz}, \mathrm{H}-6 \mathrm{~b}), 1.60\left(3 \mathrm{H}, s, \mathrm{CH}_{3}\right), 1.37\left(3 \mathrm{H}, s, \mathrm{CH}_{3}\right) .{ }^{13} \mathrm{C} \mathrm{NMR}\left(\mathrm{CDCl}_{3}, 100.6 \mathrm{MHz}\right)$ $\delta$ in ppm: $135.2(\mathrm{C}, \mathrm{Ar}), 132.1-128.1(\mathrm{CH}, \mathrm{Ar}), 111.6(\mathrm{C}), 89.5(\mathrm{CH}, \mathrm{C}-1), 78.2(\mathrm{CH}, \mathrm{C}-3), 71.2$ (CH, C-5), 70.4 (CH, C-4), $63.0\left(\mathrm{CH}_{2}, \mathrm{C}-6\right), 28.4\left(\mathrm{CH}_{3}\right), 26.8\left(\mathrm{CH}_{3}\right), 25.6(\mathrm{CH}, \mathrm{C}-2)$.

Phenyl 5-O-benzyl-2-deoxy-2-iodo-3,4-O-isopropylidene-1-thio- $\alpha$-D-altro-septanoside (20). Compound 16 (276 mg, $0.7136 \mathrm{mmol})$ was dissolved in acetonitrile $(14.3 \mathrm{ml}, 0.05 \mathrm{M})$ and cooled to $-30{ }^{\circ} \mathrm{C}$ and sodium bicarbonate was added ( $89.9 \mathrm{mg}, 1.0704 \mathrm{mmol}$ ) followed by NIS (240.8 $\mathrm{mg}, 1.0704 \mathrm{mmol})$. The reaction was controlled by TLC analysis. The reaction was stirred for 24 hours at $-10^{\circ} \mathrm{C}$, then at room temperature for 30 hours and was finally heated at $35^{\circ} \mathrm{C}$ for $24 \mathrm{~h}$. Full conversion was not reached but the reaction was quenched with a solution of sodium thiosulfate. The aqueous layer was extracted with ethyl acetate $(3 \times 20 \mathrm{ml})$, the combined organic layers were washed with water $(2 \times 20 \mathrm{ml})$, with brine $(1 \times 20 \mathrm{ml})$, dried on $\mathrm{MgSO}_{4}$, filtered and concentrated under vacuum. The reaction mixture was separated by chromatography (hexane $\rightarrow$ hexane: ethyl acetate $=1: 1)$ and compound $\mathbf{2 0}$ was obtained as a light yellow oil (45 $\mathrm{mg}, 0.0878$ mmol, 12\%. Starting material (109 $\mathrm{mg}, 0.281 \mathrm{mmol}, 40 \%)$ were also recovered.

$\mathrm{R}_{\mathrm{f}}$ (hexane:ethyl acetate=8:1): 0.38. $[\alpha]_{\mathrm{D}}{ }^{25} 156^{\circ}\left(c 0.16, \mathrm{CH}_{2} \mathrm{Cl}_{2}\right) \cdot{ }^{1} \mathrm{H}-\mathrm{NMR}^{23}\left(\mathrm{CDCl}_{3}, 400 \mathrm{MHz}\right)$ $\delta$ in ppm: $7.50-7.26(10 \mathrm{H}, m, \mathrm{Ar}), 5.56(1 \mathrm{H}, d, \mathrm{~J}=8.8 \mathrm{~Hz}, \mathrm{H}-1), 5.13(1 \mathrm{H}, t, \mathrm{~J}=8.8, \mathrm{~Hz}, \mathrm{H}-2)$, $4.73\left(1 \mathrm{H}, d, \mathrm{~J}=11.2 \mathrm{~Hz}, \mathrm{CH}_{2} \mathrm{Ph}\right), 4.70\left(1 \mathrm{H}, d, \mathrm{~J}=11.2 \mathrm{~Hz}, \mathrm{CH}_{2} \mathrm{Ph}\right), 4.65(1 \mathrm{H}, d d, \mathrm{~J}=7.6,7.6$ $\mathrm{Hz}, \mathrm{H}-3), 4.58(1 \mathrm{H}, d d, \mathrm{~J}=13.6,1.0 \mathrm{~Hz}, \mathrm{H}-6 \mathrm{a}), 4.49$ (1H, $d d, \mathrm{~J}=7.6,2.0 \mathrm{~Hz}, \mathrm{H}-4), 4.13$ (1H, $m$, $\mathrm{H}-5), 3.81(2 \mathrm{H}, d d, \mathrm{~J}=13.6,5.2 \mathrm{~Hz}, \mathrm{H}-6 \mathrm{~b}), 1.60\left(3 \mathrm{H}, s, \mathrm{CH}_{3}\right), 1.40\left(3 \mathrm{H}, s, \mathrm{CH}_{3}\right) .{ }^{13} \mathrm{C}$ NMR $\left(\mathrm{CDCl}_{3}, 100.6 \mathrm{MHz}\right) \delta$ in ppm: $138.3(\mathrm{C}, \mathrm{Ar}), 131.5-127.6(\mathrm{CH}, \mathrm{Ar}), 108.3(\mathrm{C}), 93.0(\mathrm{CH}, \mathrm{C}-1)$, 80.1 (CH, C-3), $77.8(\mathrm{CH}, \mathrm{C}-5), 76.9(\mathrm{CH}, \mathrm{C}-4), 73.5\left(\mathrm{CH}_{2}, \mathrm{CH}_{2} \mathrm{Ph}\right), 63.5\left(\mathrm{CH}_{2}, \mathrm{C}-6\right), 32.0(\mathrm{CH}$, C-2), $26.4\left(\mathrm{CH}_{3}\right), 23.9\left(\mathrm{CH}_{3}\right)$. HRMS (TOF MS ES+): calcd for $\mathrm{C}_{22} \mathrm{H}_{25} \mathrm{O}_{4} \mathrm{NaSI}(\mathrm{MNa}+)$ 535.0416; found, 535.0413. Elemental Analysis: Calculated: C: 51.57\%; H: 4.92\%; S: 6.26\%. Found: C: $51.90 \%, \mathrm{H}: 4.70 \%$, S: $6.10 \%$.

\section{(Z/E)-6-O-tert-Butyldimethylsilyl-1,2-dideoxy-3,4-O-isopropylidene-1-phenylsulfanyl-D-}

lyxo-hex-1-enitol (22). Diphenyl phenylsulfanylmethylphosphine oxide (6.488 g, $20.0 \mathrm{mmol})$ was dissolved in anhydrous THF $(20 \mathrm{ml}, 0.25 \mathrm{M})$ and $n$-BuLi $(13.1 \mathrm{ml}, 21.0 \mathrm{mmol}, 1.6 \mathrm{M}$ in hexane) was added slowly to the solution at $-30{ }^{\circ} \mathrm{C}$ and the mixture was stirred under an argon atmosphere until the occurrence of a intensive orange colour. The reaction mixture was further 
stirred for one hour at this temperature. Then a solution of 21 (1.522 g, $5.0 \mathrm{mmol})$ in anhydrous THF (10.0 ml, 0.5 M) was added. The reaction was allowed to warm up to room temperature. After full conversion (24 h) and work up, the resulting product was purified by flash chromatography (hexane:ethyl acetate $=6: 1)$ to yield compound $22(1.14 \mathrm{~g}, 2.779 \mathrm{mmol}, 56 \%)$ as a yellow oil that revealed to be an inseparable mixture of $Z / E=1 / 4$.

NMR spectral data were extracted from the diastereoisomeric mixture. $\boldsymbol{E}-22$ : $\mathrm{R}_{\mathrm{f}}$ (hexane:ethyl acetate=6:1): 0.67. ${ }^{1} \mathrm{H}-\mathrm{NMR}^{23}\left(\mathrm{CDCl}_{3}, 400 \mathrm{MHz}\right) \delta$ in ppm: 7.50-7.20 (5H, $\left.m, \mathrm{Ar}\right), 6.52(1 \mathrm{H}, d, \mathrm{~J}$ $=14.8 \mathrm{~Hz}, \mathrm{H}-1), 5.95(1 \mathrm{H}, d d, \mathrm{~J}=14.8,7.2 \mathrm{~Hz}, \mathrm{H}-2), 4.05(1 \mathrm{H}, d d, \mathrm{~J}=6.4,4.0 \mathrm{~Hz}, \mathrm{H}-4), 4.68$ $(1 \mathrm{H}, d d, \mathrm{~J}=7.2,6.4 \mathrm{~Hz}, \mathrm{H}-3), 4.13(1 \mathrm{H}, m, \mathrm{H}-5), 3.93(1 \mathrm{H}, d d, \mathrm{~J}=11.2,5.2 \mathrm{~Hz}, \mathrm{H}-6 \mathrm{a}), 3.81$ $(1 \mathrm{H}, d d, \mathrm{~J}=11.2,6.8 \mathrm{~Hz}, \mathrm{H}-6 \mathrm{~b}), 2.35(1 \mathrm{H}, d, \mathrm{~J}=5.6 \mathrm{~Hz}, \mathrm{OH}), 1.46\left(3 \mathrm{H}, s, \mathrm{CH}_{3}\right), 1.35(3 \mathrm{H}, s$, $\left.\mathrm{CH}_{3}\right), 0.92\left(9 \mathrm{H}, s, \mathrm{CH}_{3}\right), 0.12\left(6 \mathrm{H}, s, \mathrm{CH}_{3}\right) .{ }^{13} \mathrm{C} \mathrm{NMR}\left(\mathrm{CDCl}_{3}, 100.6 \mathrm{MHz}\right) \delta$ in ppm: 134.2, $(\mathrm{C}$, Ar), 130.8-126.9 (CH, Ar), 127.5 (CH, C-1), 126.9 (CH, C-2), 108.8 (C), 80.9 (CH, C-5), 80.0 $(\mathrm{CH}, \mathrm{C}-4), 78.5(\mathrm{CH}, \mathrm{C}-3), 61.6\left(\mathrm{CH}_{2}, \mathrm{C}-6\right), 27.4\left(\mathrm{CH}_{3}\right), 26.0\left(\mathrm{CH}_{3}\right), 25.2\left(\mathrm{CH}_{3}\right)$.

Spectral data is extracted from the diastereoisomeric mixture. Z-22: $\mathrm{R}_{\mathrm{f}}$ (hexane:ethyl acetate=6:1): 0.67. ${ }^{1} \mathrm{H}-\mathrm{NMR}^{23}\left(\mathrm{CDCl}_{3}, 400 \mathrm{MHz}\right) \delta$ in ppm: 7.50-7.20 (5H, $\left.m, \mathrm{Ar}\right), 6.48(1 \mathrm{H}, d, \mathrm{~J}$ $=7.8 \mathrm{~Hz}, \mathrm{H}-1), 6.04(1 \mathrm{H}, d d, \mathrm{~J}=7.8,7.2 \mathrm{~Hz}, \mathrm{H}-2), 5.17(1 \mathrm{H}, d d, \mathrm{~J}=7.2,7.2 \mathrm{~Hz}, \mathrm{H}-3), 4.32(1 \mathrm{H}$, $d d, \mathrm{~J}=7.2,2.4 \mathrm{~Hz}, \mathrm{H}-4), 4.13$ (1H, $m, \mathrm{H}-5), 3.93$ (1H, $m, \mathrm{H}-6 \mathrm{a}), 3.81$ (1H, $m, \mathrm{H}-6 \mathrm{~b}), 2.40(1 \mathrm{H}, d$, $\mathrm{J}=6.0 \mathrm{~Hz}, \mathrm{OH}), 1.46\left(3 \mathrm{H}, s, \mathrm{CH}_{3}\right), 1.35\left(3 \mathrm{H}, s, \mathrm{CH}_{3}\right), 0.92\left(9 \mathrm{H}, s, \mathrm{CH}_{3}\right), 0.12\left(6 \mathrm{H}, s, \mathrm{CH}_{3}\right)$.

\section{( $Z / E$ )-6-O-tert-Butyldimethylsilyl-5- $O$-benzyl-1,2-dideoxy-3,4-O-isopropylidene-1-}

phenylsulfanyl-D-lyxo-hex-1-enitol (23). To a suspension of sodium hydride (16 mg, 0.66 mmol) in THF, compound $22(244 \mathrm{mg}, 0.60 \mathrm{mmol})$ dissolved in freshly distilled THF $(2.4 \mathrm{ml}$, $0.25 \mathrm{M}$ ) was added at room temperature. The reaction mixture was further stirred for an hour at room temperature and subsequently freshly distilled ethyl bromide (67 $\mu 1,0.9 \mathrm{mmol})$ was slowly added. The reaction mixture was stirred overnight, and the evolution of the reaction was followed by TLC. The reaction was then quenched with a saturated ammonium chloride solution. The aqueous layer was extracted with ethyl acetate $(3 \times 20 \mathrm{ml})$, the combined organic layers were washed with water $(2 \times 20 \mathrm{ml})$, with brine $(1 \times 20 \mathrm{ml})$, dried on $\mathrm{MgSO}_{4}$, filtered and concentrated under vacuum. The mixture was purified by chromatography (hexane $\rightarrow$ hexane:ethyl acetate $=$ 1:1) and compound $\mathbf{2 3}$ was obtained as a light yellow oil (361 $\mathrm{mg}, 0.74 \mathrm{mmol}, 37 \%$ ), as an inseparable mixture of $Z / E=1 / 4$.

NMR spectral data were extracted from the diastereoisomeric mixture. $\boldsymbol{E}-23$ : $\mathrm{R}_{\mathrm{f}}$ (hexane:ethyl acetate=8:1): 0.56. ${ }^{1} \mathrm{H}-\mathrm{NMR}^{23}\left(\mathrm{CDCl}_{3}, 400 \mathrm{MHz}\right) \delta$ in ppm: 7.50-7.20 (5H, $\left.m, \mathrm{Ar}\right), 6.45(1 \mathrm{H}, d, \mathrm{~J}$ $=15.2 \mathrm{~Hz}, \mathrm{H}-1), 5.90(1 \mathrm{H}, d d, \mathrm{~J}=15.2,7.8 \mathrm{~Hz}, \mathrm{H}-2), 4.63(1 \mathrm{H}, d d, \mathrm{~J}=7.8,6.8 \mathrm{~Hz}, \mathrm{H}-3), 4.28$ $(1 \mathrm{H}, d d, \mathrm{~J}=6.8,4.0 \mathrm{~Hz}, \mathrm{H}-4), 3.74-3.64\left(3 \mathrm{H}, m, \mathrm{H}-6 \mathrm{a}, \mathrm{CH}_{2}(\mathrm{Et})\right), 3.43(1 \mathrm{H}, d d, \mathrm{~J}=9.2,7.2 \mathrm{~Hz}$, $\mathrm{H}-6 \mathrm{~b}), 3.26(1 \mathrm{H}, m, \mathrm{H}-5), 1.46\left(3 \mathrm{H}, s, \mathrm{CH}_{3}\right), 1.35\left(3 \mathrm{H}, s, \mathrm{CH}_{3}\right), 1.21-1.14\left(3 \mathrm{H}, m, \mathrm{CH}_{3}\right), 0.92$ $\left(9 \mathrm{H}, s, \mathrm{CH}_{3}\right), 0.12\left(6 \mathrm{H}, s, \mathrm{CH}_{3}\right) .{ }^{13} \mathrm{C} \mathrm{NMR}\left(\mathrm{CDCl}_{3}, 100.6 \mathrm{MHz}\right) \delta$ in ppm: $134.5(\mathrm{C}, \mathrm{Ar}), 130.2-$ 127.0 (CH, Ar), 126.9 (CH, C-1), 126.6 (CH, C-2), 109.1 (C), 78.4 (CH, C-5), 77.6 (CH, C-3), $76.8(\mathrm{CH}, \mathrm{C}-4), 66.7\left(\mathrm{CH}_{2}\right), 62.6\left(\mathrm{CH}_{2}, \mathrm{C}-6\right), 29.8\left(\mathrm{CH}_{3}\right), 27.4\left(\mathrm{CH}_{3}\right), 26.0\left(\mathrm{CH}_{3}\right), 25.2\left(\mathrm{CH}_{3}\right) . Z-$ 23: $\mathrm{R}_{\mathrm{f}}$ (hexane/AcOEt=8/1): 0.55. ${ }^{1} \mathrm{H}-\mathrm{NMR}^{23}\left(\mathrm{CDCl}_{3}, 400 \mathrm{MHz}\right) \delta$ in ppm: 7.50-7.20 $(5 \mathrm{H}, \mathrm{m}$, Ar), $6.48(1 \mathrm{H}, d, \mathrm{~J}=7.8 \mathrm{~Hz}, \mathrm{H}-1), 6.04(1 \mathrm{H}, d d, \mathrm{~J}=7.8,7.2 \mathrm{~Hz}, \mathrm{H}-2), 5.17(1 \mathrm{H}, d d, \mathrm{~J}=7.2,7.2$ 
Hz, H-3), 4.32 (1H, dd, J = 7.2, 2.4 Hz, H-4), 3.74-3.26 (5H, m, H-5, H-6a, H-6b, CH $(\mathrm{Et})), 1.46$ $\left(3 \mathrm{H}, s, \mathrm{CH}_{3}\right), 1.35\left(3 \mathrm{H}, s, \mathrm{CH}_{3}\right), 1.21-1.14\left(3 \mathrm{H}, m, \mathrm{CH}_{3}\right), 0.92\left(9 \mathrm{H}, s, \mathrm{CH}_{3}\right), 0.12\left(6 \mathrm{H}, s, \mathrm{CH}_{3}\right)$.

\section{(Z/E)-1,2-Dideoxy-5-O-ethyl-3,4-O-isopropylidene-1-phenylsulfanyl-D-lyxo-hex-1-enitol}

(24). Compound 23 (150 mg, $0.34 \mathrm{mmol})$ was dissolved in THF $(3.0 \mathrm{ml})$ and tetrabutylammonium fluoride $(118.3 \mathrm{mg}, 0.38 \mathrm{mmol})$ was added to the solution. The reaction mixture was stirred at room temperature and the reaction was monitored by TLC. After one hour the reaction was quenched with a saturated sodium carbonate solution. The aqueous layer was extracted with ethyl acetate $(3 \times 20 \mathrm{ml})$, the combined organic layers were washed with water $(2 \times 20 \mathrm{ml})$, with brine $(1 \times 20 \mathrm{ml})$ dried on $\mathrm{MgSO}_{4}$, filtered and concentrated under vacuum. The mixture was separated by chromatography (hexane $\rightarrow$ hexane:ethyl acetate $=1: 1$ ) and compound 24 was obtained as a light yellow oil (89 $\mathrm{mg}, 0.277 \mathrm{mmol}, 81 \%$ as an inseparable mixture of $Z / E$ $=1 / 4$.

NMR spectral data were extracted from the diastereoisomeric mixture. $\boldsymbol{E}-\mathbf{2 4}$ : $\mathrm{R}_{\mathrm{f}}$ (hexane:ethyl acetate=4:1): 0.51. ${ }^{1} \mathrm{H}-\mathrm{NMR}^{23}\left(\mathrm{CDCl}_{3}, 400 \mathrm{MHz}\right) \delta$ in ppm: 7.40-7.24 $(5 \mathrm{H}, m, \mathrm{Ar}), 6.54(1 \mathrm{H}, d$, $\mathrm{J}=15.2 \mathrm{~Hz}, \mathrm{H}-1), 5.96(1 \mathrm{H}, d d, \mathrm{~J}=15.2,6.4 \mathrm{~Hz}, \mathrm{H}-2), 4.78(1 \mathrm{H}, d d, \mathrm{~J}=6.4,5.6 \mathrm{~Hz}, \mathrm{H}-3), 4.06$ $(1 \mathrm{H}, d d, \mathrm{~J}=8.8,5.6 \mathrm{~Hz}, \mathrm{H}-4), 3.86-3.41\left(5 \mathrm{H}, m, \mathrm{H}-5, \mathrm{H}-6 \mathrm{a}, \mathrm{H}-6 \mathrm{~b}, \mathrm{CH}_{2}(\mathrm{Et}), 2.46(1 \mathrm{H}, d, \mathrm{~J}=5.2\right.$ $\mathrm{Hz}, \mathrm{OH}), 1.45\left(3 \mathrm{H}, s, \mathrm{CH}_{3}\right), 1.35\left(3 \mathrm{H}, s, \mathrm{CH}_{3}\right), 1.22-1.04\left(3 \mathrm{H}, m, \mathrm{CH}_{3}\right) .{ }^{13} \mathrm{C} \mathrm{NMR}\left(\mathrm{CDCl}_{3}, 100.6\right.$ $\mathrm{MHz}) \delta$ in ppm: 134.5 (C, Ar), 130.2-127.0 (CH, Ar), 126.9 (CH, C-1), 126.6 (CH, C-2), 109.1 (C), $78.4(\mathrm{CH}, \mathrm{C}-5), 77.6(\mathrm{CH}, \mathrm{C}-4), 76.8(\mathrm{CH}, \mathrm{C}-3), 69.7\left(\mathrm{CH}_{2}\right), 61.6\left(\mathrm{CH}_{2}, \mathrm{C}-6\right), 27.4\left(\mathrm{CH}_{3}\right)$, $26.0\left(\mathrm{CH}_{3}\right), 25.2\left(\mathrm{CH}_{3}\right)$. ZZ-24: $\mathrm{R}_{\mathrm{f}}$ (hexane:ethyl acetate=4:1): 0.51. ${ }^{1} \mathrm{H}_{-N M R}{ }^{23}\left(\mathrm{CDCl}_{3}, 400\right.$ $\mathrm{MHz}) \delta$ in ppm: 7.50-7.20 (5H, $m, \mathrm{Ar}), 6.48(1 \mathrm{H}, d, \mathrm{~J}=7.8 \mathrm{~Hz}, \mathrm{H}-1), 6.04(1 \mathrm{H}, d d, \mathrm{~J}=7.8,7.2$ $\mathrm{Hz}, \mathrm{H}-2), 5.17(1 \mathrm{H}, d d, \mathrm{~J}=7.2,7.2 \mathrm{~Hz}, \mathrm{H}-3), 4.32(1 \mathrm{H}, d d, \mathrm{~J}=7.2,2.4 \mathrm{~Hz}, \mathrm{H}-4), 3.74-3.26(5 \mathrm{H}$, $\left.m, \mathrm{H}-5, \mathrm{H}-6 \mathrm{a}, \mathrm{H}-6 \mathrm{~b}, \mathrm{CH}_{2}(\mathrm{Et})\right), 2.51(1 \mathrm{H}, d, \mathrm{~J}=5.2 \mathrm{~Hz}, \mathrm{OH}), 1.46\left(3 \mathrm{H}, s, \mathrm{CH}_{3}\right), 1.35(3 \mathrm{H}, s$, $\left.\mathrm{CH}_{3}\right), 1.21-1.14\left(3 \mathrm{H}, m, \mathrm{CH}_{3}\right)$.

2-Deoxy-5-O-ethyl-3,4-O-isopopylidene-2-iodo- $\beta$-D-galacto-septanose (26a) and 2-deoxy-5$\boldsymbol{O}$-ethyl-3,4- $\boldsymbol{O}$-isopopylidene-2-iodo- $\alpha$-D-galacto-septanose (26b). Compound 24 (89 mg, $0.277 \mathrm{mmol})$ was dissolved in acetonitrile $(5.5 \mathrm{ml}, 0.05 \mathrm{M})$ and cooled to $-30{ }^{\circ} \mathrm{C}$, and then sodium bicarbonate ( $35 \mathrm{mg}, 0.415 \mathrm{mmol}$ ) and NIS (93.3 $\mathrm{mg}, 0.415 \mathrm{mmol}$ ) were added. The reaction was stirred overnight at $-10{ }^{\circ} \mathrm{C}$, then at room temperature for 30 hours and finally heated at $35{ }^{\circ} \mathrm{C}$ for 24 hours. The reaction was then quenched with the addition of a saturated solution of sodium thiosulphate. The aqueous layer was extracted with ethyl acetate $(3 \times 20 \mathrm{ml})$, the combined organic layers were washed with water $(2 \times 20 \mathrm{ml})$, with brine $(1 \times 20 \mathrm{ml})$ dried on $\mathrm{MgSO}_{4}$, filtered and concentrated under vacuum. The mixture was separated by chromatography (hexane $\rightarrow$ hexane:ethyl acetate $=1: 1$ ) and compounds 26a,b were obtained as an inseparable mixture as a light yellow oil (23 mg, $0.099 \mathrm{mmol}, 36 \%$ ). Starting material $Z \mathbf{Z}-24$ (29 mg, $0.088 \mathrm{mmol}, 32 \%$ ) was also recovered.

$\mathbf{R}_{\mathrm{f}}$ (hexane:ethyl acetate=4:1): 0.37. NMR spectral data were extracted from the isomeric mixture. 26a: ${ }^{1} \mathrm{H}-\mathrm{NMR}^{23}\left(\mathrm{CDCl}_{3}, 400 \mathrm{MHz}\right) \delta$ in ppm: $5.48(1 \mathrm{H}, d, \mathrm{~J}=1.2 \mathrm{~Hz}, \mathrm{H}-1), 4.72(1 \mathrm{H}$, $d d, \mathrm{~J}=10.0,8.0 \mathrm{~Hz}, \mathrm{H}-3), 4.25(1 \mathrm{H}, d d, \mathrm{~J}=8.0,7.2 \mathrm{~Hz}, \mathrm{H}-4), 4.18(1 \mathrm{H}, d d, \mathrm{~J}=10.0,1.2 \mathrm{~Hz}, \mathrm{H}-$ 2), $3.95 \mathrm{1H}, d d, \mathrm{~J}=13.2,9.6 \mathrm{~Hz}, \mathrm{H}-6 \mathrm{a}), 3.57$ (1H, $d d, \mathrm{~J}=13.2,2.0 \mathrm{~Hz}, \mathrm{H}-6 \mathrm{~b}), 3.47$ (1H, $m, \mathrm{H}-5)$, 
$1.51\left(3 \mathrm{H}, s, \mathrm{CH}_{3}\right), 1.39\left(3 \mathrm{H}, s, \mathrm{CH}_{3}\right), 1.33-1.18\left(3 \mathrm{H}, m, \mathrm{CH}_{3}\right) .{ }^{13} \mathrm{C} \mathrm{NMR}\left(\mathrm{CDCl}_{3}, 100.6 \mathrm{MHz}\right) \delta$ in ppm: $108.2(\mathrm{C}), 96.9(\mathrm{CH}, \mathrm{C}-1), 80.4(\mathrm{CH}, \mathrm{C}-4), 78.8(\mathrm{CH}, \mathrm{C}-5), 76.5(\mathrm{CH}, \mathrm{C}-3), 66.1\left(\mathrm{CH}_{2}\right.$, $\left.\mathrm{CH}_{2} \mathrm{Me}\right), 60.7\left(\mathrm{CH}_{2}, \mathrm{C}-6\right), 35.4(\mathrm{CH}, \mathrm{C}-2), 27.7\left(\mathrm{CH}_{3}\right), 24.9\left(\mathrm{CH}_{3}\right), 15.6\left(\mathrm{CH}_{3}, \mathrm{Et}\right) .26 \mathbf{b}:{ }^{1} \mathrm{H}$ NMR $\left(\mathrm{CDCl}_{3}, 400 \mathrm{MHz}\right) \delta$ in ppm: $5.41(1 \mathrm{H}, d, \mathrm{~J}=8.0 \mathrm{~Hz}, \mathrm{H}-1), 4.41(1 \mathrm{H}, d d, \mathrm{~J}=11.6,7.2 \mathrm{~Hz}$, $\mathrm{H}-3), 4.33$ (1H, $m, \mathrm{H}-6 \mathrm{a}), 4.32$ (1H, $m, \mathrm{H}-4), 4.10(1 \mathrm{H}, d d, \mathrm{~J}=11.6,8.0 \mathrm{~Hz}, \mathrm{H}-2), 3.78-3.74(2 \mathrm{H}$, $\left.m, \mathrm{H}-5, \mathrm{CH}_{2}(\mathrm{Et})\right), 3.59-3.54\left(1 \mathrm{H}, m, \mathrm{CH}_{2}(\mathrm{Et})\right), 3.48-3.45(1 \mathrm{H}, m, \mathrm{H}-6 \mathrm{~b}), 1.51\left(3 \mathrm{H}, s, \mathrm{CH}_{3}\right), 1.39$ $\left(3 \mathrm{H}, s, \mathrm{CH}_{3}\right), 1.33-1.18\left(3 \mathrm{H}, m, \mathrm{CH}_{3}\right) .{ }^{13} \mathrm{C} \mathrm{NMR}\left(\mathrm{CDCl}_{3}, 100.6 \mathrm{MHz}\right) \delta$ in ppm: $109.1(\mathrm{C}), 98.1$ (CH, C-1), 78.5 (CH, C-4), 78.1 (CH, C-5), $77.0(\mathrm{CH}, \mathrm{C}-3), 67.0\left(\mathrm{CH}_{2}, \mathrm{CH}_{2} \mathrm{Me}\right), 62.0\left(\mathrm{CH}_{2}, \mathrm{C}-\right.$ 6), $32.5(\mathrm{CH}, \mathrm{C}-2), 27.5\left(\mathrm{CH}_{3}\right), 24.5\left(\mathrm{CH}_{3}\right), 15.8\left(\mathrm{CH}_{3}, \mathrm{Et}\right)$. HRMS (TOF MS ES+): calcd for $\mathrm{C}_{11} \mathrm{H}_{19} \mathrm{O}_{5} \mathrm{NaI}(\mathrm{MNa}+)$ 381.0175; found, 381.0180. Elemental Analysis: Calculated: C, 36.89\%; H, 5.35\%. Found: C: $37.55 \%$, H: 5.47\%.

\section{Acknowledgement}

Financial support from DGESIC CTQ2005-03124-BQU (Ministerio de Ciencia y Tecnología, Spain) is acknowledged. We are also grateful to the Servei de Recursos Cientifics (URV) for its technical assistence.

\section{References and Footnontes}

1. Pakulski, Z. Pol. J. Chem. 1996, 70, 667.

2. Castro, S.; Duff, M.; Snyder, N. L.; Morton, M.; Kumar, C. V.; Peczuh, M. W. Org. Biomol. Chem. 2005, 3, 3869.

3. Tauss, A.; Steiner, A. J.; Stütz, A. E.; Tarling, C. A.; Whiters, S. G.; Wrodnigg, T. M. Tetrahedron:Asymmetry 2006, 17, 234.

4. (a) Martínez-Mayorga, K.; Medina-Franco, J. L.; Mari, S., Cañada, F. J.; Rodríguez-García, E.; Vogel, P.; Li, H.; Blériot, P.; Sinaÿ, P.; Jiménez-Barbero, J. Eur. J. Org. Chem. 2004, 4119. (b) Morís-Varas, F.; Qian, X.-H.; Wong, C.-H. J. Am. Chem. Soc. 1996, 118, 7647.

5. Benner, S. A.; Sismour, A. M. Nat. Rev. Genet. 2005, 6, 533.

6. (a) Peczuh, M. W.; Snyder, N. L. Tetrahedron Lett. 2003, 44, 4057. (b) Peczuh, M. W.; Snyder, N. L.; Fyvie, W. S. Carbohydr. Res. 2004, 339, 1163.

7. Alcázar, E.; Pletcher, J. M.; McDonald, F. W. Org. Lett. 2004, 6, 3877.

8. (a) DeMatteo, M. P.; Snyder, N. L.; Morton, M.; Baldisseri, D. M.; Hadad, C. M.; Peczuh, M. W. J. Org. Chem. 2005, 70, 24. (b) Fyvie, W. S.; Morton, M.; Peczuh, M. W. Carbohydr. Res. 2004, 339, 2363.

9. Castro, S.; Fyvie, W. S.; Hatcher, S. A.; Peczuh, M. W. Org. Lett. 2005, 16, 4709.

10. (a) Castro, S.; Peczuh, M. W. J. Org. Chem. 2005, 70, 3312. (b) McAuliffe, J. C.; Hindsgaul, O. Synlett 1998, 307. 
11. (a) Rodríguez, M. A.; Boutureira, O.; Arnés, X.; Matheu, M. I.; Díaz, Y.; Castillón, S. J. Org. Chem. 2005, 70, 10297. (b) Arnés, X.; Díaz, Y.; Castillón, S. Synlett 2003, 2143.

12. Thio-glycosides are useful glycosyl donors, see for instance: (a) Oscarson, S. In Carbohydrates in Chemistry and Biology, Ernst, B.; Hart, G. W.; Sinaÿ, P. Eds., Wiley: Weinheim, 2000; Part I, Vol. I, p 93. (b) Garegg, P. J. Adv. Carbohydr. Chem. Biochem. 1997, 52, 172.

13. 2-Deoxy-thioglycosides have recently been used as glycosyl donors in a solid-phase-assisted synthesis of 2-deoxyconjugates: Jaunzems, J.; Hofer, E.; Jesberger, M.; Sourkouni-Argirusi, G.; Kirschning, A. Angew. Chem. Int. Ed. 2003, 42, 1166.

14. Boutureira, O. ; Rodríguez, M. A.; Matheu, M. I.; Díaz, Y.; Castillón, S. Org. Lett. 2006, 8, 673.

15. (a) Rousseau, G.; Homsi, F. Chem. Soc. Rev. 1997, 453. (b) Simonot, B.; Rousseau, G. J. Org. Chem.1994, 59, 5912.

16. Brunel, Y.; Rousseau, G. J. Org. Chem. 1996, 61, 5793.

17. Bhattacharya, A. K.; Thyagarajan, G. Chem. Rev. 1981, 81, 415.

18. Barlett, P. A.; Mayerion, J. J. Am. Chem. Soc. 1978, 100, 3950.

19. Lipshutz, B. H.; Tirado, R. J. Org. Chem. 1994, 59, 8307.

20. Aucagne, V.; Tatibouët, A.; Rollin, P. Tetrahedron 2004, 60, 1817.

21. (a) Landais, Y.; Panchenault, D. Synlett 1995, 1191. (b) Bravo, F.; Castillón, S. Eur. J. Org. Chem. 2001, 507.(b) Stork, G.; Kahn, M. Tetrahedron Lett. 1983, 24, 3951. (c) Houk, K. N.; Moses, S. R.; Wu, Y.-D.; Rondan, N. G.; Jäger, V.; Schohe, R.; Fronczek, F. R. J. Am. Chem. Soc. 1984, 106, 3880. (d) Halter, J.; Strassner, T.; Houk, K. N. J. Am. Chem. Soc. 1997, 119, 8031.

22. Bravo, F. Castillón, S. Eur. J. Org. Chem. 2001, 507.

23. For clarity, hydrogen and carbon atoms have been numbered according to the respective alkene starting material. 